\title{
Estrategia de los productores del sur de Yucatán para insertarse en la economía de mercado
}

\author{
José Antonio Ávila Dorantes
}

Universidad Autónoma Chapingo-Centro Regional Universitario Península de Yucatán. Exhacienda Temozón Norte, S/N. Domicilio Conocido municipio de Mérida, Yucatán. Tel. 9999240054.

Autor para correspondencia: aviladorantes@hotmail.com.

\section{Resumen}

El propósito de este artículo es destacar el caso de los productores de una comunidad del sur de Yucatán, que siguen una estrategia basada en la obtención de ingresos dentro de su unidad de producción, que generalmente, los arraiga a su comunidad. El objetivo es caracterizar la agricultura familiar de la comunidad de Yáaxhom, Yucatán, y analizar el comportamiento de los productores, a través del ingreso monetario, para resolver su problema económico cotidiano. Para la caracterización de la comunidad se siguió el método general para el estudio multilateral de la agricultura, mientras que para la obtención de los ingresos monetarios netos se adecuó la teoría económica convencional de ingresos y costos a las unidades de producción que no se comportan como empresas, y se obtuvo una muestra estadística de la comunidad. Los resultados sugieren que la integración de un mercado cercano, disponibilidad de recursos naturales, organización de los productores, apoyos gubernamentales y desarrollo de policultivos, han hecho que los productores no tengan interés en abandonar sus unidades de producción. El origen del ingreso dentro de la unidad de producción es diversificado y fluye durante prácticamente todo el año. La promoción por parte de las autoridades de estrategias similares en otras regiones puede inhibir la emigración campo-ciudad.

Palabras clave: agricultura familiar, economía convencional, estrategias de producción, fuentes de ingreso.

Recibido: enero de 2021

Aceptado: febrero de 2021 
Una de las teorías muy aceptadas durante el siglo pasado fue el papel de la agricultura en el crecimiento económico. En las primeras etapas del crecimiento, la agricultura debe transferir recursos a los otros sectores de la producción, como mano de obra, bienes-salario o capital, para apoyarlas en el despegue económico (Schultz, 1956; Rostov, 1961; Kuznets, 1968). Posteriormente, desde la década de los sesenta del siglo pasado, se promovió en la academia (Hayek, 1994; Gordon, 1996), que el crecimiento económico de los países se basara en un modelo que se inclinara más al libre mercado y apertura comercial, por lo que el Estado se iría retirando y su lugar sería ocupado paulatinamente por la iniciativa privada. La inclinación a una mayor apertura comercial condujo a la economía mundial a un proceso de globalización.

El crecimiento económico de buena parte del mundo siguió estas estrategias. Por ejemplo, en los Estados Unidos de Norteamérica, en el período 1920-1949, más de 50 millones de habitantes dejaron la agricultura para dedicarse a otras actividades (Schultz, 1956). En México, en el siglo pasado, en el período de auge del sector agrícola, 1942-1961, éste transfirió al resto de la economía recursos de mano de obra, a través de la emigración campo-ciudad, y recursos de capital a través del efecto combinado del régimen fiscal, el sistema bancario, y el mecanismo de precios (Reyes et al., 1974).

En lo que va del presente siglo, López (2000) afirma que en el sector rural del país sigue viviendo una cuarta parte de la población y que para el período 1991-2003, la población dedicada a las actividades agropecuarias disminuyó $21 \%$ al pasar de 9.8 a 7.7 millones de personas. Últimamente y al nivel de región, en Yucatán, se han presentado migraciones de los pueblos milperos a polos de desarrollo como Mérida y Progreso y más recientemente a las zonas turísticas de la Riviera Maya y de Cancún en Quintana Roo (Fraga, 2015).

Sin embargo, estas estrategias que han predominado desde mediados del siglo pasado hasta nuestros días, han sido incapaces de absorber la mano de obra que se fue liberando de la economía, entre ellos, la de los productores medianos y pequeños de la agricultura. Por ejemplo, en México, la participación del trabajo, dentro del producto interno bruto (PIB), durante el período 1995-2015, ha ido disminuyendo tanto en los sectores de bienes comerciales como en los no comerciales (Zamaniego, 2017). Entre las razones principales de esta disminución parecen estar el avance de la tecnología, que sustituye trabajo a favor de capital, en el proceso de globalización, que implica el uso de tecnología de punta, obreros altamente calificados, cadenas de valor y otros (Zamaniego, 2017).

Las estrategias deben cambiar. El sector secundario no ha podido absorber la mano de obra desplazada en la agricultura y ha ido a engrosar las filas de la informalidad en el sector terciario, con salarios muy deprimidos y sin seguridad social, así como la emigración fundamentalmente a los Estados Unidos de América, Ávila et al. (2008). El flujo de mano de obra de la agricultura a la industria y los servicios debe inhibirse y si es posible, cambiar su sentido e impulsar actividades que estimulen el empleo en el campo.

Los productores de campo elaboran una variedad de bienes que venden o autoconsumen (agrícolas, pecuarios, forestales), también utilizan diversos productos y recursos de su unidad de producción para el disfrute familiar (plantas medicinales, de ornato, para la construcción) y otros como la captura de carbono y la proporción de oxígeno a la sociedad, que con frecuencia no se contabilizan en la formación del ingreso de estos productores. Esta producción diversa, se desarrolla en México, 
en $76 \%$ de las unidades familiares agrícolas (UFA) y son las que generan más empleo, cuidan el medio ambiente, y contribuyen fuertemente a la soberanía alimentaria en América Latina y El Caribe (Leporati et al., 2014; Salcedo et al., 2014).

De lo expresado, se desprende la necesidad de analizar las actividades que se desarrollan en el campo, que estimulan y permiten la permanencia de los productores rurales y sus descendientes, e inhibir el flujo migratorio entre campo-ciudad. Para esto, es fundamental analizar estrategias que estén efectuando las UFA en cuanto a su ingreso y su distribución durante el año, que les permita a las personas que viven en el campo, en edad de buscar empleo, a tener más alternativas al tomar sus decisiones de dónde trabajar.

\section{Justificación}

Desde el punto de vista de la teoría económica convencional, una persona que desea entrar al mercado de trabajo puede dedicar su tiempo disponible entre trabajo y descanso (Nicholson, 2005), el descanso incluye todas las actividades que quiera desarrollar, y por las cuales no obtiene ingreso. Los productores rurales dedican su tiempo disponible entre trabajar dentro de su unidad de producción, fuera de ella, o utilizarlo en descanso, hasta el punto en que el valor del producto marginal de cada una de ellas se iguale (Ahearn et al., 2006). Hay diferentes razones por las cuales los productores rurales abandonan su unidad de producción; desde el punto de vista económico, la explicación se encuentra en que el valor de su producto marginal esperado es mayor trabajando fuera que dentro de su unidad de producción (Nicholson, 2005).

La teoría económica convencional enseña que la utilidad o satisfacción de una persona está representado por lo que gasta en bienes y servicios. Este gasto es menor o igual a su ingreso, pues la diferencia, si la hay, la puede ahorrar (ya que el ahorro representa gasto futuro) (Samuelson y Nordhaus, 2003; Romer, 2018). Hay una relación directa entre el ingreso y el ahorro.

El ahorro aumenta, si aumenta el ingreso y viceversa. Para las familias con escasos ingresos por cabeza, que forma parte de una de las nueve variables que considera el Consejo Nacional de Evaluación de la Política de Desarrollo Social (CONEVAL, 2018) para la medición de la pobreza, el ingreso es con frecuencia, insuficiente para cubrir el consumo, por lo que se puede considerar a su ahorro como insignificante o nulo. Esto significa que todo su ingreso se utiliza en consumo. Desde el punto de vista económico, el aumento en consumo significa aumento de su satisfacción, por lo que, aumentar su ingreso significa aumentar su satisfacción.

El objetivo fue caracterizar la agricultura familiar de una comunidad, así como analizar el comportamiento de los productores, a través del ingreso monetario, para resolver su problema económico cotidiano, y que les permite mantenerse en su unidad de producción.

\section{Metodología}

El municipio de Oxkutzcab tiene una altura promedio de $33 \mathrm{msnm}$. Está a $80 \mathrm{~km}$ de la Ciudad de Mérida, Yucatán en dirección sureste. La mayor parte del territorio municipal es plano. Sin embargo, se encuentra surcado por una serranía en dirección este-oeste, en la cual está ubicada la comisaría de Yáaxhom, a una distancia de $10 \mathrm{~km}$ al sureste de la cabecera municipal. Una de las interpretaciones sobre el significado de la palabra Yáaxhom es 'Primera Hondonada' (yáax, 
primero y k'om, hondonada) (Bastarrachea y Canto, 2007). La población es de alrededor de 300 habitantes. Aproximadamente $90 \%$ de la comunidad cuenta con agua potable en sus viviendas. La electricidad es un servicio relativamente nuevo, y que beneficia a aproximadamente $75 \%$ de la población.

El clima en la región es cálido-subhúmedo, presenta una temperatura media anual de $26.3{ }^{\circ} \mathrm{C}$ y un rango de $26{ }^{\circ} \mathrm{C}-28{ }^{\circ} \mathrm{C}$ con lluvias en los meses de mayo y junio fundamentalmente. Cuando se interrumpen se presenta la sequía de medio verano, con un rango de precipitación de 1 000-1 100 $\mathrm{mm}$. La humedad relativa en el mes de marzo es $66 \%$ y en el mes de diciembre es del $89 \%$. Los vientos dominantes proceden del sureste. Los suelos son fértiles para uso agrícola (Duch, 1988). La profundidad del agua de los pozos que se usan para riego está entre 60 y $70 \mathrm{~m}$ y se cultiva fundamentalmente cítricos (limón, 84\%; naranja, 67\%; mandarina, 33\%), aguacate, 75\%, maíz, $33 \%$ y otros cultivos, $58 \%$ (como mango, pepino, calabaza, mamey, coco, toronja, zapote, saramuyo, guanábana). Debido a la costumbre de intercalar en sus frutales otros cultivos como los anuales y las hortalizas, y de sembrar diversos frutales en forma asociada, los productores de la localidad de Yaax-hom trabajan sus parcelas de una manera intensiva.

Para la caracterización de la comunidad se siguió con frecuencia la metodología que contempla cinco niveles de investigación: el ámbito productivo, los procesos laborales, las unidades productivas, los agentes dominantes, y los sectores sociales del Centro Regional Universitario de la Península de Yucatán (CRUPY) de la Universidad Autónoma Chapingo (UACH), (Macossay, 1991).

Se realizó una fase de recorrido físico por la zona de estudio y se estableció contacto con la autoridad municipal, comisariado y diversos productores líderes. Luego, una fase de campo durante el año 2015, con equipos de estudiantes del CRUPY. Esta fase incluyó dos etapas: 1) revisiones metodológicas y documentales referenciales sobre factores de clima, orografía y socioeconomía; y 2) estancias en la comunidad, recogiendo y verificando información a través de cuestionario, libreta de campo y conviviendo y apoyando en las actividades cotidianas de las familias.

Se obtuvo un tamaño de muestra aleatoria (12) de las 60 familias de Yáaxhom, por el método del muestreo irrestricto aleatorio (MIA) (Scheaffer et al., 2007), a los cuales se les aplicó entrevistas de acuerdo con un cuestionario modificado que con frecuencia utiliza el CRUPY. Luego se vació la información en hojas de cálculo del programa Excell para su análisis posterior.

Partiendo de una unidad de producción que funciona como empresa, el ingreso neto se calcularía (Samuelson y Nordhaus, 2004): IT $=P Q \quad$ (1) $\quad \mathrm{CT}=\mathrm{CV}+\mathrm{CF}$ (2), G= IT-CT (3). En la cual: $\mathrm{IT}=$ ingreso total; $\mathrm{P}=$ precio de venta; $\mathrm{Q}=$ producción $; \mathrm{CT}=$ costo total $\mathrm{CV}=$ costo variable; $\mathrm{CF}=$ costo fijo; $\mathrm{G}=$ ingreso neto (ganancia). Para que este concepto se adapte al funcionamiento de las UFA (no buscan utilidades), las cuales están relacionadas al mercado de insumos y productos, ya sea adquiriendo cuando menos algún insumo, y vendiendo parte o toda su Q, el CV se puede dividir en CV1 y CV2. El CV1 representa los costos desembolsados por la UFA por variar la producción (Q). Pueden representar mano de obra asalariada e insumos como: agua, fertilizantes, mejoradores, plaguicidas y otros, pero que representan erogaciones para el jefe de la unidad productiva. El CV2 los mismos insumos, pero que no representan gastos. Pueden representar mano de obra familiar $\mathrm{u}$ otro insumo como agua o fertilizante, que hayan sido donados por alguna institución pública o privada. 
El mismo procedimiento se sigue para el CF. Se puede dividir en CF1 y CF2. Los primeros representan las erogaciones que tuvo que hacer la UFR para estar en condiciones de producir y cuyo monto es independiente del volumen de producción. Los CF2 representan lo mismo que el anterior, solamente que no representaron erogaciones, pues fueron donados por alguna institución pública o privada.

Los CT de las UFR se obtienen a partir de la ecuación (2): CT= CV1+CV2+CF1+CF2 (4), Llamando CT1= CV1+CF1 (5); CT2= CV2+CF2 (5a). Desde luego, CT1 es una función de CT2. Su valor depende del que asuma CT2, pues los productores harán todo lo posible por producir con los costos desembolsados más bajos. $\mathrm{CT} 1=\mathrm{F}(\mathrm{CT} 2)$; si CT2=0; CT1= CT; si CT2= CT; CT1=0, Sustituyendo las ecuaciones (5 y 5a) en la (3); G= IT-(CT1+CT2); G+CT2= IT-CT1; llamando $\mathrm{G}^{*}=\mathrm{G}+\mathrm{CT} 2 ; \mathrm{G}^{*}=\mathrm{IT}-\mathrm{CT} 1(6)$.

Si se considera que $C T 1=C V 1$, pues $C F 1=0$; la ecuación (6) se convierte en $\mathrm{G}^{*}=\mathrm{IT}-\mathrm{CV} 1 . \mathrm{G}^{*}$ representa el ingreso neto que obtienen los productores de las UFR por trabajar dentro de ella. Si la $G^{*}$ se divide entre el número de jornales de mano de obra familiar (LF) que se utilizó en el proceso de producción, se tendría un estimado del ingreso familiar por jornal (WF) que obtiene la UFR por trabajar en su unidad de producción. Desde luego, la comparación de WF con el salario del mercado (W) enriquece el análisis del comportamiento del productor entre abandonar o no su unidad de producción.

Los ingresos netos que se presentan en el cuadro correspondiente están expresados a precios de mercado de 2015 y en forma ascendente de acuerdo con el ingreso monetario obtenido. En la obtención del ingreso neto se consideran los precios que recibe y los que paga el productor. No toda la producción se convierte en dinero, pues una parte se usa en autoconsumo familiar; sin embargo, en este trabajo, la producción que no entra al mercado es cuantificada en valor a través de su costo de oportunidad (se deja de ganar en el mejor uso alternativo del recurso en cuestión).

Para tener una idea de la magnitud, se calcula alrededor de $\$ 25587.00$ el ingreso de una persona que ganara el salario mínimo de 2015 (\$70.10 diario) durante un año. Para llevar estos valores al 2020, se utiliza una inflación acumulada de 20\%, de acuerdo con el índice nacional de precios al productor del sector primario (INEGI, 2020) y un salario mínimo de $\$ 123.22$ de acuerdo con la Comisión Nacional de Salarios Mínimos (CONASAMI, 2020). La muestra de población de Yáaxhom está seccionada en 12 estratos.

\section{Características de las UFA}

Ámbito productivo: Yáaxhom está situado en una hondonada de La Sierrita Puuc. El suelo es más fértil en Yucatán, con una temperatura benigna durante el año, con pequeñas variaciones y con buenas lluvias en cuanto a cantidad y distribución, y disponibilidad de agua para riego a base de pozos profundos), la zona ha permitido el desarrollo de una amplia variedad de plantas y animales.

Buena parte de estas bondades de la región son el resultado de un apoyo decidido de parte del Estado, a través de la implementación de diferentes proyectos productivos (establecimiento de unidades de riego), fundamentalmente frutícolas y del estímulo a la organización de los productores para el uso y conservación de la infraestructura de riego. Además, el Estado ha impulsado, en menor escala, otros proyectos productivos, sobre todo de aves, para los solares de las casas. 
La edad del jefe de familia, en su mayoría el padre, de la población encuestada, es en promedio de 52 años y son quienes generalmente trabajan en la unidad de producción. Las familias no suelen ser tan numerosas; el número de hijos fluctúa alrededor de 4 personas. En relación, al nivel de estudios de la población encuestada, se destaca que 50\% concluyó la primaria, $17 \%$ la secundaria y $8 \%$ terminó la preparatoria y alcanzó un nivel de educación superior (licenciatura, ingeniería, etc.). Sin embargo, $17 \%$ no concluyó sus estudios de primaria y $8 \%$ es analfabeta. En relación con el idioma, $92 \%$ de los productores encuestados son bilingües, hablan castilla y maya. Las personas de mayor edad son las que mejor dominan la lengua indígena y las nuevas generaciones la dominan en diferentes niveles.

Contrariamente a otras regiones (Yúnez-Naude, 2001; Lozano, 2003; Gordillo, 2018), en Yáaxhom hay reducida migración de la población a otras entidades o al extranjero. Un $50 \%$ de los jefes de familia no viven en la comunidad, pero sí en la cabecera municipal de los hijos, $72 \%$ se encuentra viviendo en el municipio $97 \%$ lo hace en Yucatán y $98 \%$ en la península, mientras que la emigración al extranjero únicamente representa $2 \%$.

\section{Procesos laborales}

En la actualidad, en las parcelas de los productores predominan los frutales, fundamentalmente cítricos (limón, naranja y mandarina) y aguacate. Hay tendencia generalizada a tener diversos frutales mezclados con algunas plantas forestales en la misma parcela (sobre todo cedro y caoba). Sumado a lo anterior, con frecuencia se siembran hortalizas (tomate, chile) o cultivos anuales (maíz y frijol) en algunos claros de la parcela.

Las actividades que se derivan de la atención a la unidad de producción (incluyendo el solar de la casa) estimulan la presencia del productor o su sustituto (familiar o asalariado) en ella. Por ejemplo, una hectárea de frutales requiere 176 jornales al año, destacando el riego (104), la cosecha (24), los deshierbes (20), poda (10) y aplicación de herbicida (9), mientras que las otras actividades requieren 9 jornales (elaboración de pocetas, plantación, aplicación de insecticida y aplicación de fertilizante). El productor, mientras vigila el riego, ocupa su tiempo en realizar parte de las actividades anteriores.

El uso de fertilizantes orgánicos (abono animal), químicos, de herbicidadas e insecticidas, está bastante extendido entre los productores, y no así la de los biofertilizantes y bioplaguicidas, aunque son conocidos. El uso masivo de estos insumos causa daños al ambiente, aun cuando no son percibidos actualmente; además, el manejo de los envases vacíos no es el adecuado en toda la cadena, empezando con el productor.

Los productores disponen de terrenos ejidales fundamentalmente, mientras que la propiedad privada coexiste en menor grado. Existe un mercado de derechos de usufructo de las parcelas, los cuales pueden ser por tiempo indeterminado. El tamaño de la unidad de producción es en promedio de 4.31 hectáreas, con 3.54 en producción; sin embargo, la mayor parte de la unidad de producción está fraccionada. Si se tomara en consideración el costo de la infraestructura de riego y su conservación (el cual con frecuencia es apoyado por las autoridades), el valor del mercado de la tierra que posee (son tierras ejidales en su gran mayoría), más los instrumentos de trabajo que poseen (coas, machetes, cosechadoras manuales, azadón, hacha, entre otros), el grado de capitalización de las UFA es alto. 
Una cuarta parte de las familias de la comunidad se dedican también a la atención de aves, fundamentalmente gallinas, mientras que casi una quinta parte cría y engorda cerdos. Ambas actividades las realizan en los solares de las casas. Los corrales y gallineros son pequeños y simples construcciones, cuyo material, en ocasiones, es proporcionado por las autoridades. Los trabajos de atención y mantenimiento son efectuados fundamentalmente por algún familiar (la cónyuge) durante todo el año, y pueden llegar a representar hasta 90 días de trabajo de 8 horas.

Las actividades forestales prácticamente no son tomadas en cuenta por los productores, ya que su aprovechamiento es circunstancial, escaso y para autoconsumo. Además de las plantas maderables, como caoba y cedro, se aprovechan otros como el jabín (Piscidia piscipula) y el Dzidzilché (Gymnopodium-floribundum) para leña, poleo o menta (Mentha pulegium) de tipo medicinal.

La población de animales de la región sigue siendo importante en cantidad y variedad. Desde la década de los ochenta se menciona, que en fauna, está representada por: mono araña (Ateles geoffroyi), conejo (Sylvilagus floridanus), mapache (Procyon sp.), venado (Odocoileus virginianus), iguanas (Ctenosaurus sp.), serpientes (Crotalus terrificus), tortugas (Terrapene carolina yucatana), chachalaca (Ortalis vetula), chivicoyo (Colinus virginianus) y pavo de monte (Meleagris ocellata) (Pérez, 1984).

\section{Las unidades de producción}

Los productores están organizados por unidades de riego (pozos), y cada una de ellas aglutina en promedio a más de 30 usuarios. Su organización permite que los apoyos externos (gubernamentales o no) lleguen al conocimiento de todos los integrantes de la unidad; los cuales, a través de asambleas expresan su opinión y sus puntos de vista son tomados en cuenta. Sin embargo, en la compra de insumos y venta del producto, se manejan de forma individual.

Se consideraron seis posibles fuentes de ingreso monetario: el valor de la producción agrícola, pecuaria, forestal, del solar (agrícola y pecuaria), de los recursos forestales de recolección, y de las artesanías. Sin embargo, la fortaleza de estos ingresos proviene de la producción agrícola y del solar, mientras que las otras 4 fuentes están poco desarrolladas, o no se identificaron, como el caso de las artesanías. El ingreso agrícola se distribuye durante todo el año, pues la cosecha del producto es variada. El limón todo el año, sobre todo en los períodos mayo-septiembre y octubre-abril; el aguacate todo el año, sobre todo marzo-mayo y octubre-febrero, la naranja temprana septiembrediciembre, naranja tardía, noviembre-abril y la mandarina, octubre-febrero. A los ingresos que se derivan de los productos frutícolas, se les suma los que provienen de los cultivos anuales y las hortalizas.

La producción forestal está poco trabajada y puede representar una alternativa para aumentar los ingresos de la unidad de producción, la venta de la caoba y el cedro es prácticamente nulo, existen diversas variedades de árboles en la región que podrían impulsarse con fines comerciales (Pérez, 1984), por ejemplo, el chukum (Havardia albicans), de cuya corteza se extraen los taninos que se usan en los acabados de la industria de la construcción regional. En los ingresos que provienen de otra fuente diferente a la unidad de producción, destacan los trabajos del jefe de familia y en menor grado los apoyos gubernamentales (Prospera, Setenta y Más) y los apoyos familiares, mientras que no se identificaron ingresos provenientes de remesas. 


\section{Los agentes dominantes y los sectores sociales}

Los agentes políticos y económicos dominantes se expresan a través de las diferentes instancias gubernamentales en que está estructurado la forma de gobierno federal, estatal y municipal, por una parte y por las diferentes estructuras de mercado presentes en los productos de compraventa y en los mercados de capitales, por otra. Los productores de Yáaxhom, como en las demás localidades del país, se encuentran influidos en forma considerable por los apoyos oficiales en salud, educación y otros, que a través de las diferentes instancias gubernamentales y de los partidos políticos, les llegan en forma de programas.

En la venta de productos y compra de insumos, los productores se comportan como oferentes y demandantes tomadores de precios, como si estuvieran en mercados competitivos, y se enfrentan a demandantes y oferentes que actúan en forma oligopólica, por lo que permiten, como vendedores, que una parte considerable del valor agregado de los productos y que paga el consumidor final, pase a manos de estos compradores (intermediarios independientes o ligados a empresas regionales y nacionales) y deja sin aprovechar, como compradores, los menores precios de los insumos que puede adquirir, si organizara sus compras en forma masiva. En la infraestructura se presenta de nuevo el funcionamiento desventajoso del mercado. Los productores requieren innovarse para adaptarse a esta situación (Bosc, 2016), ampliar las funciones de su organización que en la actualidad giran alrededor del manejo del agua, e incursionar con su asociación en los otros mercados que forman su cadena productiva.

\section{Estructura del ingreso}

Estructura del ingreso de la unidad de producción: Esta variable está formada por la suma de los valores de la producción de las actividades agrícolas, pecuarias, forestales, del solar y de los productos forestales de recolección, que se desarrollan dentro de la unidad de producción (Cuadro 1). Existe una variedad de árboles frutales, cuya venta permite a los productores, tener ingresos distribuidos durante el año, de acuerdo con la temporada de cosecha y también de los tipos de frutales plantados. Por predominar estos árboles en las unidades de producción, en promedio general, del valor de la producción agrícola, la mayoría de lo cosechado se orienta a la venta (más de $95 \%$ ), quedando para el autoconsumo menos de $5 \%$.

Sin embargo, al nivel de estratos, el autoconsumo puede representar hasta $21 \%$ en $8 \%$ de los productores, pues aun cuando las parcelas están orientadas al comercio, 33\% de los productores siembran maíz en sus unidades de producción, de los cuales, $75 \%$ de ellos es para autoconsumo, mientras que $25 \%$ (de 33\%) además del maíz siembra en forma intercalada frijol y calabaza. Casi la totalidad de lo vendido es transportada al mercado de la cabecera municipal (Oxkutzcab).

Es notorio que únicamente $33 \%$ de los entrevistados reporte actividades pecuarias. Éstas son realizadas generalmente por la pareja del productor y por algunos de los otros dependientes de la familia. Estas labores se realizan generalmente en el solar de la casa, y para los que lo practican, representa un ingreso en efectivo importante (\$18 622.00) y más de $40 \%$ de lo que se obtiene es para la venta. Si se toman en cuenta las actividades tanto pecuarias como agrícolas, éstas se realizan en $75 \%$ de los solares de los entrevistados. 
Aproximadamente $42 \%$ de las familias de Yáaxhom utilizan con frecuencia las plantas forestales que han cultivado o crecido en forma silvestre, (para enriquecer la fuente de sus abastecimientos y aumentar el ingreso monetario (Cuadro 1), a través de su uso doméstico: árboles para combustible en el hogar; para techos de las casas; medicinales; para espantar moscos, y ocasionalmente, maderables como el cedro y la caoba.

\section{Estructura del ingreso obtenido fuera de la unidad de producción}

Una parte de los productores de la comunidad obtienen ingresos monetarios trabajando fuera de sus unidades de producción. De esta manera, tienen una actividad principal y otra secundaria. Sin embargo, en ocasiones, puede revertirse la situación, y la actividad fuera de la parcela sea más importante que la que desarrolla dentro de ella. En el caso de Yáaxhom, 42\% aproximado de jefes de familia laboran fuera de su parcela. Se identificaron jefes de familia que desarrollan actividades diversas como taxistas, empleados en la iniciativa privada, burócratas, poceros, y jornaleros.

Estos resultados confirman lo expresado por Tomaselli (2016), que afirma que la superación de la pobreza no depende únicamente de la capacidad de los hogares de generar ingresos; a partir, de la unidad de producción o de la comunidad, sino de todos los lugares en que pueda encontrar ocupación sin necesidad de abandonar su residencia habitual. Los resultados también enriquecen el análisis de esta comunidad, en su proceso de descampesiniciación, dentro de la teoría de la economía campesina (Calva, 1988). Existen subsidios del gobierno; sin embargo, los confiables y periódicos son: Prospera y 70 y Más, el primero es el más extendido. El $42 \%$ de las familias del poblado cuenta con este tipo de apoyo gubernamental. Estructura del ingreso neto total.

Cuadro 1. Estructura del ingreso neto total. Pesos y participaciones.

\begin{tabular}{ccccccccccccc}
\hline Estrato & 1 & 2 & 3 & 4 & 5 & 6 & 7 & 8 & 9 & 10 & $\begin{array}{c}\text { Ingreso } \\
\text { total } \\
\text { (relativo) }\end{array}$ & $\begin{array}{c}\text { Ingreso } \\
\text { total }(\$)\end{array}$ \\
\hline 1 & 1 & 0 & 0 & 0 & 0 & 0 & 0 & 0 & 0 & 0 & 1 & 13395 \\
2 & 1 & 0 & 0 & 0 & 0 & 0 & 0 & 0 & 0 & 0 & 1 & 56588 \\
3 & 0.061 & 0 & 0 & 0.681 & 0.007 & 0 & 0 & 0.251 & 0 & 0 & 1 & 57445 \\
4 & 0.005 & 0 & 0 & 0.01 & 0.003 & 0 & 0.93 & 0.062 & 0 & 0 & 1 & 77386 \\
5 & 0.088 & 0.086 & 0 & 0 & 0 & 0 & 0.826 & 0 & 0 & 0 & 1 & 116228 \\
6 & 0.645 & 0 & 0 & 0 & 0 & 0 & 0 & 0 & 0 & 0.355 & 1 & 123915 \\
7 & 0.882 & 0.116 & 0 & 0 & 0.002 & 0 & 0 & 0 & 0 & 0 & 1 & 125700 \\
8 & 0.344 & 0 & 0 & 0.286 & 0 & 0 & 0.332 & 0.038 & 0 & 0 & 1 & 126675 \\
9 & 0.69 & 0 & 0 & 0.31 & 0 & 0 & 0 & 0 & 0 & 0 & 1 & 165248 \\
10 & 0.554 & 0 & 0 & 0.386 & 0.004 & 0 & 0 & 0.056 & 0 & 0 & 1 & 171136 \\
11 & 0.781 & 0.151 & 0 & 0.009 & 0.001 & 0 & 0.059 & 0 & 0 & 0 & 1 & 175217 \\
12 & 0.183 & 0.114 & 0 & 0.087 & 0 & 0 & 0.591 & 0.026 & 0 & 0 & 1 & 206764 \\
\hline
\end{tabular}

Ingreso neto total $=1+2+3++9+10.1=$ valor de la producción agrícola; $2=$ valor de la producción pecuaria; $3=$ valor de la producción forestal; 4= valor de la producción del solar; 5= valor de recursos forestales de recolección; $6=$ valor de producción de artesanías; $7=$ ingreso del jefe de familia por trabajar fuera de la unidad de producción; 8= sumatoria de subsidios del Estado (Prospera + 70 y más); 9= Remesas; 10= apoyos familiares (no remesas). 
En la formación del ingreso neto total, destaca el valor de la producción agrícola en casi todos los casos. Un 58\% de los jefes de familia obtienen ingresos agrícolas provenientes de su unidad de producción que representan más de $50 \%$ de su ingreso neto total. El porcentaje aumenta al $67 \%$ de las familias, si aquél representa $30 \%$ del total. Otra variable importante la constituyen los ingresos que obtiene por trabajar fuera de su unidad de producción. Cuando esta variable se presenta (en $42 \%$ de los jefes de familia), en la mayoría de los casos es más de $30 \%$ del ingreso neto total.

Al relacionar estos ingresos con las actividades que desarrollan dentro de su unidad de producción, se encuentra que estos jefes de familia usan con frecuencia mano de obra alquilada. Los resultados del trabajo de campo y de las encuestas sugieren que parte de estos jefes de familia (25\%) requieren mano de obra alquilada en sus unidades de producción, pues su actividad fuera de ellas se ha vuelto la principal en términos de ingresos netos relativos, mientras que al restante $17 \%$ no alcanza lo que produce la unidad de producción y deben complementarlos con los que obtengan fuera de ella.

Si la estructura del valor de la producción generada en la unidad de producción se trabaja con base en su destino, resulta que está enfocada fundamentalmente para abastecer al mercado y en menor proporción al autoconsumo. Sin embargo, esta variable no es despreciable, ya que en 33\% de las familias, el autoconsumo representa como mínimo 14\%, y como máximo $32 \%$ del ingreso neto total. Si se analiza el Cuadro 1 en cuanto al origen, $75 \%$ de los jefes de familia obtienen ingresos mayores en su unidad de producción que fuera de ella.

Si se comparan los ingresos netos totales obtenidos por los productores, con el correspondiente al salario mínimo de un año (2015), 8\% de los productores (estrato 1) no alcanza a obtener un salario mínimo como ingreso neto, en sus diferentes actividades a las que se dedica. Los dos estratos siguientes ( 2 y 3), obtienen ingresos entre dos y tres salarios mínimos. El cuarto nivel (8\%) apenas alcanza los tres salarios. Los tres siguientes estratos (5, 6 y 7) que, representan $25 \%$ de los productores, obtienen ingresos que fluctúan entre los 4 salarios. Los cinco estratos mayores restantes (42\%), alcanzan ingresos que fluctúan entre cinco hasta poco más de ocho salarios mínimos al año (Cuadro 1 y 2).

Resulta mayor, a excepción del primer estrato, el ingreso neto diario (WF) que obtiene el productor en sus diferentes actividades a las que se dedica, que el que habría obtenido con el salario promedio rural que se pagaba en 2015 en la zona (W), suponiendo que encontrara trabajo. Esto significa, desde el punto de vista del ingreso, que la estrategia que siguen los productores de Yáaxhom es mejor que la alternativa de emplear su recurso de mano de obra de otra manera. Si estos 'salarios' se extrapolan al año 2020, los resultados se mantienen cualitativamente, pero cuantitativamente se deteriora el WF, como consecuencia de un mayor aumento en el salario mínimo (76\%), en el W (25\% más de 120), mientras que los precios al productor aumentaron $20 \%$ (Cuadro 2).

Cuadro 2. Cálculo de diferentes 'salarios' para las UFA de Yáaxhom. Pesos corrientes.

\begin{tabular}{ccccccccccc}
\hline \multicolumn{1}{c}{ Estrato } & $\begin{array}{c}\text { Ingreso } \\
\text { total }\end{array}$ & $\begin{array}{c}\text { Ingreso } \\
\text { diario }\end{array}$ & $\begin{array}{c}\text { ingreso/sal } \\
\text { mínimo }\end{array}$ & $\begin{array}{c}\text { ingreso/sal } \\
\text { promedio }\end{array}$ & & $\begin{array}{c}\text { Ingreso } \\
\text { total }\end{array}$ & $\begin{array}{c}\text { Ingreso } \\
\text { diario }\end{array}$ & $\begin{array}{c}\text { ingreso/sal } \\
\text { mínimo }\end{array}$ & $\begin{array}{c}\text { ingreso/sal } \\
\text { promedio }\end{array}$ \\
\hline 1 & 13395 & 37 & 0.5 & 0.3 & & 16074 & 44 & 0.4 & 0.3 \\
2 & 56588 & 155 & 2.2 & 1.3 & & 67905.6 & 186 & 1.5 & 1.2 \\
\hline
\end{tabular}




\begin{tabular}{|c|c|c|c|c|c|c|c|c|}
\hline \multicolumn{5}{|c|}{2015} & \multicolumn{4}{|c|}{2020} \\
\hline Estrato & $\begin{array}{c}\text { Ingreso } \\
\text { total }\end{array}$ & $\begin{array}{c}\text { Ingreso } \\
\text { diario }\end{array}$ & $\begin{array}{l}\text { ingreso/sal } \\
\text { mínimo }\end{array}$ & $\begin{array}{c}\text { ingreso/sal } \\
\text { promedio }\end{array}$ & $\begin{array}{c}\text { Ingreso } \\
\text { total }\end{array}$ & $\begin{array}{c}\text { Ingreso } \\
\text { diario }\end{array}$ & $\begin{array}{c}\text { ingreso/sal } \\
\text { mínimo }\end{array}$ & $\begin{array}{c}\text { ingreso/sal } \\
\text { promedio }\end{array}$ \\
\hline 3 & 57445 & 157 & 2.2 & 1.3 & 68934 & 189 & 1.5 & 1.3 \\
\hline 4 & 77386 & 212 & 3 & 1.8 & 92863.2 & 254 & 2.1 & 1.7 \\
\hline 5 & 116228 & 318 & 4.5 & 2.7 & 139473.6 & 382 & 3.1 & 2.5 \\
\hline 6 & 123915 & 339 & 4.8 & 2.8 & 148698 & 407 & 3.3 & 2.7 \\
\hline 7 & 125700 & 344 & 4.9 & 2.9 & 150840 & 413 & 3.4 & 2.8 \\
\hline 8 & 126675 & 347 & 5 & 2.9 & 152010 & 416 & 3.4 & 2.8 \\
\hline 9 & 165248 & 453 & 6.5 & 3.8 & 198297.6 & 543 & 4.4 & 3.6 \\
\hline 10 & 171136 & 469 & 6.7 & 3.9 & 205363.2 & 563 & 4.6 & 3.8 \\
\hline 11 & 175217 & 480 & 6.8 & 4 & 210260.4 & 576 & 4.7 & 3.8 \\
\hline 12 & 206764 & 566 & 8.1 & 4.7 & 248116.8 & 680 & 5.5 & 4.5 \\
\hline
\end{tabular}

Cálculos directos en base a: salario mínimo abril 2015 zona A: 70.10; salario promedio de la zona en 2015: 120; salario mínimo enero 2020 resto del país: 123.22; inflación de enero 2015 a diciembre 2020: 20\% (99-79) según INPP del sector primario; salario promedio de la zona en 2020: $\$ 150.00$.

\section{Estrategia basada en el origen del ingreso}

Para aprovechar las ventajas de las economías de escala, el Estado impulsó en la década de los sesenta del siglo pasado, el desarrollo del sur de la entidad yucateca, con un programa que le llamó El Plan Chaac. Consistía, entre otras cosas, en sembrar 5000 ha de naranja dulce en siete municipios, entre ellos Oxkutzcab (Ávila, 2015). En los hechos, los agricultores adaptaron la tecnología propuesta, resultando en una que integraba la moderna con la tradicional.

Ya funcionando El Plan Chaac, los productores sembraban diversos frutales y con frecuencia también, cultivos anuales, hortalizas y criaban diversos animales. La región sur de la entidad se consolidó de un paisaje planeado de monocultivo, a otro de policultivo, el cual, ya venía realizándose con los esfuerzos individuales de las familias de la región. Esta experiencia permeó en diversas comunidades, entre ellas, Yáaxhom.

La estrategia de policultivo seguida por las UFA de Yáaxhom, les permite obtener ingresos de diversas fuentes dentro de su unidad de producción y prácticamente, durante todo el año. Al dedicarse a los policultivos, sus costos unitarios de producción en que incurren deben prorratearse entre el producto de la diversidad de actividades que realizan, por lo que el ingreso neto es mayor al que se obtendría si hubiera practicado el monocultivo; además, por autoemplearse, y emplear a los otros miembros de su familia, no tiene que pagar los salarios correspondientes, lo que aumenta nuevamente su ingreso neto, vía disminución de costos erogados. Por otra parte, las poliactividades agrícolas, pecuarias y forestales realizadas por las UFA de Yáaxhom, proporcionan empleo y biodiversidad, por lo que apoyan decididamente el combate al desempleo y a la contaminación ambiental. La captura de carbono es otro producto de la UFA, el cual aumentaría su ingreso neto, si se valorara a precio de mercado este servicio ambiental. 
La mayoría de las UFA de Yáaxhom (75\%) obtienen ingresos netos mayores dentro de su unidad de producción que, trabajando fuera de ella, por lo que tienen fuertes incentivos para considerarla su actividad principal, y permanecer en ella. El restante $25 \%$ de las UFA, al obtener ingresos mayores al trabajar fuera que dentro de la unidad de producción, desestimula su permanencia en la UFA y alienta a dejársela a los otros miembros del hogar, a alquilar mano de obra, a rentar o hasta vender su unidad de producción.

Las UFA siguen siendo importantes en el sector agropecuario. Representan todavía, 76\% de las unidades de producción en México y al nivel De América Latina y el Caribe: 81\% de las explotaciones agrícolas; provee, a nivel país, de $27 \%$ al $67 \%$ del total de la producción alimentaria, ocupa entre el $12 \%$ y $67 \%$ de la superficie agropecuaria, y genera entre $57 \%$ y $77 \%$ del empleo agrícola en la Región (FAO-BID, 2007; FAO, 2012).

\section{Conclusiones}

La integración de una cercana localización y de fácil acceso al mercado, disponibilidad de adecuados recursos naturales, organización de los productores en el manejo del agua, apoyos gubernamentales frecuentes, espíritu de superación y poliactividades en sus UFA, han hecho que los productores de Yáaxhom tengan poco interés en abandonar sus unidades de producción, aún para aquéllos que habitan fuera, pero cercanamente a la comunidad.

La orientación de la producción al mercado, la diversidad de árboles frutales, con preponderancia de algunos, intercalados con árboles forestales, cultivos anuales u hortalizas, le dan a la unidad productiva un paisaje que tiende a lo agroforestal y estimula la presencia del productor, a través del requerimiento de diversas actividades, predominando el riego.

El origen del ingreso dentro de la unidad de producción es diversificado y disponible durante prácticamente todo el año; en la integración de este ingreso, influye en buena medida la que proviene del solar de la casa, como parte integral de la unidad de producción y en la cual destaca el trabajo de la mujer. En la estructura del ingreso que el productor obtiene fuera de la unidad de producción, destaca el que se deriva de su salario como trabajador. Cuando el ingreso que obtiene es mayor que el que obtiene dentro de la unidad de producción, tiene que contratar mano de obra y ésta pasa a ser una actividad secundaria.

La falta de organización en la compra de insumos y venta del producto, que se manifiesta en aumento de costos y en la pulverización de los montos ofrecidos a los productores en el mercado (al comportarse como tomadores de precios), frente a vendedores y compradores con poder económico, logístico y de informática (que establecen los precios), aumentan los costos en que incurren y deprimen los ingresos que reciben.

\section{Literatura citada}

Ávila, J. 2015. Desarrollo del sur de Yucatán. El Plan Chaac: 1960-1990. Universidad Autónoma Chapingo (UACH). Primera edición. Mérida, Yucatán, México. 146 p.

Ávila, J.; Puyana, A. y Romero, J. 2008. Presente y futuro del sector agrícola mexicano en el contexto del TLCAN. Colegio de México-Universidad Autónoma Chapingo. Primera edición. Ciudad de México, México. 385 p. 
Bastarrachea, M. J. y Canto, R. J. 2007. Diccionario maya popular. Maya-español, español-maya. Academia de la Lengua Maya de Yucatán, AC. Segunda reimpresión. Mérida, Yucatán, México. 490 p.

Bosc, P.; Piraux, M. y Dulcire, M. 2016. Contribuir con la innovación, las políticas y la democracia local. In: Las agriculturas familiares y los mundos del futuro. Sourisseau (Ed.). Primera edición. Agencia Francesa para el Desarrollo (AFD) e Instituto Interamericano de Cooperación para la Agricultura (IICA). San José Costa Rica. 163-186 pp.

Calva, J. 1988. Los campesinos y su devenir en las economías de mercado. Siglo XXI. Primera edición. Ciudad de México, México. 664 p.

CONASAMI. 2020. Tabla de salarios mínimos vigentes. <https://www.gob.mx/cms/uploads/ attachment/file/525061/Tabla_de_salarios_m_nmos_vigentes_apartir_del_01_de_enero_de_ 2020.pdf>.

CONASAMI. 2020. Tabla de salarios mínimos vigentes. <https://www.gob.mx/cms/uploads/ attachment/file/525061/Tabla_de_salarios_m_nmos_vigentes_apartir_del_01_de_enero_de_ 2020.pdf>.

CONEVAL. 2018. Anexo único de los lineamientos y criterios generales para la definición, identificación y medición de la pobreza. https://www.coneval.org.mx/Normateca/ Documents/ANEXO-Lineamientos-DOF26 2018.pdf $>$.

Duch, J. 1988. La conformación territorial del estado de Yucatán. Universidad Autónoma Chapingo (UACH). Primera edición. Texcoco, Estado de México. 427 p.

Fraga, J. y Arias, L. 2015. Milperos o turisteros: opciones laborales de los jóvenes maya-yucatecos contemporáneos. México. Teoría y Praxis. Número especial: 174-196. Doi:Https://doi.org/10.22403/UQROOMX/TYPNE2015/09.

Gordon, R. 1996. Macroeconomía. Compañía Editorial Continental, SA de CV. Primera edición. Ciudad de México, México. 615 p.

Hayek, F. 1994. La desnacionalización del dinero. España: Planeta-De Agostini. Primera edición. Viscaya, España. 160 p.

INEGI. 2020. Índice nacional de precios al productor. https://www.inegi.org.mx/temas/ inpp/\#Tabulados>.

INEGI. 2020. Índice nacional de precios al productor. https://www.inegi.org.mx/temas/ inpp/\#Tabulados.

Kuznets, S. 1968. El crecimiento económico y la contribución de la agricultura: notas sobre mediciones. In: la agricultura en el desarrollo económico. Problemas de medición en el sector agrícola. Eicher, C. y Witt, L. (Eds.). Primera edición. Editorial Limusa Wiley, SA. Ciudad de México, México. 119-138 pp.

Leporati, M.; Salcedo, S.; Jara, B.; Boero, V. y Muñoz, M. 2014. La agricultura familiar en cifras. In: agricultura familiar en América Latina y el Caribe: recomendaciones de Política. Salcedo, S. y Guzmán, L. (Eds). Primera edición. FAO. Santiago, Chile. 35-56 pp.

López, F. 2000. El sector agropecuario en México. Subsecretaría de Agricultura-Secretaría de Agricultura, Ganadería, Desarrollo Rural, Pesca y Alimentación, (SAGARPA). Primera edición. Ciudad de México. 26 p. https://www.odepa.gob.cl/odepaweb/publicaciones/ NodosExcelencia/SectorAgropecuarioMexico.pdf.

Macossay, M. 1991. Método general para el estudio multilateral de la agricultura. Centro Regional Universitario de la Península de Yucatán, Universidad Autónoma Chapingo (UACH). Manuscrito. Mérida, Yucatán, México. 95 p. http://www.pued.unam.mx/export/sites/ default/publicaciones/16/Propuestas_estrategicas.pdf. 
Nicholson, W. 2005. Microeconomic theory, basic principles and extensions. Thompson SouthWestern. Nine editions. USA. 740 p.

Pérez, J. 1984. Caracterización y utilización de la clasificación maya de suelos en el municipio de Oxkutzcab, Yucatán. Departamento de Suelos, Universidad Autónoma Chapingo (UACH). Tesis de Licenciatura. Texcoco, Estado de México, México. 144 p.

Reyes, S; Stavenhagen, R; Ekstein, S. y Ballesteros, J. 1974. Estructura agraria y desarrollo agrícola en México. Fondo de Cultura Económica (FCE). Primera edición. Ciudad de México, México. 1174 p.

Rostov, W. 1961. Las etapas del crecimiento económico. Fondo de Cultura Económica (FCE). Primera edición. Ciudad de México, México. 220 p.

Salcedo, S. y Guzmán, L. 2014. El concepto de agricultura familiar en América Latina y El Caribe. In: agricultura familiar en América Latina y el Caribe: recomendaciones de política. Salcedo, S. y Guzmán, L. (Eds.). Primera edición. FAO. Santiago, Chile.17-34 p.

Scheaffer, R.; Mendenhall, W. y Ott, L. 2007. Elementos de muestreo. Editorial Paraninfo. Séptima edición. Madrid, España. 462 p.

https://books.google.com.mx/books/about/Elementos_de_muestreo.html?id=o50wlT7hceoC.

Schultz, T. 1956. La organización económica de la agricultura. Primera edición (español). Fernández y Fernández, R. Fondo de Cultura Económica (FCE). Ciudad de México, México. 432 p.

Tomaselli, A. 2016. Pobreza, vulnerabilidad y oportunidades en los territorios funcionales chilenos (1992-2002). In: trampas territoriales de pobreza, desigualdad y baja movilidad social: los casos de Chile, México y Perú. Bebbington, A.; Escobal, J.; Soloaga, I. y Tomaselli, A. (Eds.). Primera edición. Centro de Estudios Espinosa Yglesias, AC. Ciudad de México, México. 57-124 pp.

Zamaniego, N. 2017. Distribución funcional del ingreso. In: propuestas estratégicas para el desarrollo 2019-2024. Colección Informe del Desarrollo en México. Cordera, R. y Provencio, E. (Coords.). Avance. Coordinación de Humanidades Programa Universitario de Estudios del Desarrollo. Universidad Nacional Autónoma de México (UNAM). Ciudad de México, México. 41-52 pp. http://www.pued.unam.mx/export/sites/default/ publicaciones/ 16/Propuestas_estrategicas.pdf. 